Research Article

\title{
Double grain boundary configurations on graphite surfaces
}

\author{
A. Capasso ${ }^{\text {a, * }}$, F. Muñoz-Rojas ${ }^{\text {b }}$, B. Gupta ${ }^{\text {c, d }}$, N. Motta ${ }^{\text {c, d }}$, E. Placidi c, e, *** \\ a International Iberian Nanotechnology Laboratory, 4715-330, Braga, Portugal \\ b Centro de Investigación en Ciencia e Ingeniería de Materiales and Escuela de Física, Universidad de Costa Rica, San José, 11501, Costa Rica \\ ' School of Chemistry and Physics, Queensland University of Technology, 2 George St, 4000, Brisbane, Australia \\ d Centre for Materials Science, Queensland University of Technology, 2 George Street, Brisbane, QLD, 4000, Australia \\ e Department of Physics, Sapienza University of Rome, P.le Aldo Moro 2, 00185, Rome, Italy
}

\section{A R T I C L E I N F O}

\section{Article history:}

Received 29 May 2020

Received in revised form

30 July 2020

Accepted 17 August 2020

Available online 26 August 2020

\section{Keywords:}

Graphene

Highly oriented pyrolytic graphite (HOPG)

Scanning tunneling microscopy

Misorientation angle

Defects

Dislocations

\begin{abstract}
A B S T R A C T
We investigated the atomic structure of different kinds of grain boundaries on highly oriented pyrolytic graphite (HOPG) by scanning tunneling microscopy. We categorized several grain boundary configurations as a function of the misorientation angle between the adjacent grains, highlighting the occurrence of double grain boundaries (i.e., systems of two grain boundaries separated by a nanometric-scale inner region with specific atomic arrangement) for misorientation angles in the range $22^{\circ}-32^{\circ}$. By using Molecular Dynamics simulations, we analyzed the structure and energy stability of single and double grain boundaries according to the misorientation angle. The experimental evidence is corroborated by Molecular Dynamics results and total energy calculations, which found a comparable stability between single and double grain boundaries for the same misorientation angle range. Our combination of experimental measurements and theoretical calculation extends the understanding of the structural configuration of large angle grain boundaries beyond the range of misorientation angles reported to date.
\end{abstract}

() 2020 Elsevier Ltd. All rights reserved.

\section{Introduction}

Grain boundaries (GB) are interfaces between domains of a material with different crystallographic orientations. They usually form during the growth of a material with polycrystalline structure. In highly oriented pyrolytic graphite (HOPG) and graphene, its monolayer counterpart, GBs consists of structural defects in the atomic lattice and, as such, they can affect the electron properties in their vicinity, for instance by decreasing the charge mobility via electron scattering [1,2] DFT calculations showed that, when crossing a GB, the charge carriers can undergo high transparency or perfect reflection over a large energy range in accordance to the GB structure [3]. Scanning tunneling microscopy and spectroscopy (STM/STS) helped identifying and detailing the atomic structure [4] and electronic properties of GBs in HOPG [5,6]. By correlating scanning probe and transport measurements with transmission electron microscopy, it was shown that in certain conditions GBs

\footnotetext{
* Corresponding author.

** Corresponding author. Department of Physics, Sapienza University of Rome, P.le Aldo Moro 2, 00185, Rome, Italy.

E-mail addresses: andrea.capasso@inl.int (A. Capasso), ernesto.placidi@ uniroma1.it (E. Placidi).
}

can weaken the mechanical strength of graphene while leaving unaltered the electrical properties [7]. Combined STM/STS and theoretical studies unveiled the correlation between the atomic configuration of different GBs and the localized electronic states in their vicinity [8]. Such a correlation is highly dependent on the misorientation angle between adjacent grains, defined as $\theta=\mid \theta_{R}+$ $\theta_{L} \mid$, where $\theta_{R}$ and $\theta_{L}$ are the angles referred to the GB axis [3]. For small $\theta$, the spectra exhibit a pair of electron-hole symmetric Van Hove singularities peaks close to the charge neutrality point, while at larger $\theta$ the electron-hole symmetry is broken [8]. Diery et al. [9] were able to provide a consistent picture of the experimentally observed trends in Ref. [10,11]. They concluded that tilted GBs introduce non-dispersive high frequency phonon modes in polycrystalline graphene, strongly localized in the core of the GBs, while no such localized modes are found in the case of the translational boundary models [9]. In principle, these transport properties might be artificially tuned via "GB engineering" to develop nanoscale electronic devices in graphene and HOPG [12]. In fact, a precise engineering of periodic GBs with tunable transport gaps would allow the control of charge carriers without introducing bulk bandgaps in otherwise semi-metallic graphene. More in general, GB engineering in two-dimensional materials might introduce properties such as magnetism [13], one-dimensional conduction 
[14], suppression of charge carrier scattering [15] or changes in fracture mechanic [16]. For these reasons, widening the knowledge about the types of GBs that can be found in graphene/graphite surfaces, while understanding their likelihood (from an energetic standpoint) and structural properties, can be of fundamental importance, also in view of future technological possibilities.

In graphene's hexagonal lattice, the two simplest and more stable GB configurations, typically found for low $\theta$, are made of regular arrays of $(1,0)$ and $(1,1)$ basic dislocations [3]. The former configuration is made of adjacent heptagon and pentagon, and the latter by heptagon and pentagon separated by two hexagons. In HOPG and polycrystalline graphene, GBs are found throughout the surface, appearing as periodic structures with identifiable patterns of pentagonal or heptagonal rings of bright spots, when observed by scanning probe microscopy $[10,12,17,18]$. The GBs observed by STM can be rather complex, including single and double grain boundaries (SGB and DGB) $[1,5,10,12,17,18]$. The presence of DGB have also been found to strengthen graphene [19,20]. From a geometrical and energetic standpoint, the simplest configuration for a SGB is based on regular arrays of $(1,0)$ dislocations, but a SGB with this configuration cannot form for $\theta>21.8^{\circ}$ [3].Therefore, the complexity of the SGB structure is expected to increase at higher $\theta$ [3,13]. For $21.8^{\circ}<\theta<32.2^{\circ}$, no simple structures can be used to build a SGB, and thus the formation of a DGB can occur within this range.

In the present work, we analyze several GBs measured by STM on HOPG with unprecedented clarity. By using a theoretical simulation we model the structure of several kinds of complex GBs. We have been able to obtain a snapshot of both stable and non-stable configurations in graphite, showing the occurrence of both ordered and non-ordered DGBs. In view of prospective applications, DGB could be particularly relevant for the formation of nanoscale waveguides for charge carriers, which have been theoretically posited [21,22]. Our results can thus aid the identification of DGBs in certain grain-orientation conditions and thus set the basis for an investigation into the electronic transport properties of these structures.

\section{Experimental and computational methods}

Samples of HOPG (NT-MDT, ZYH grade) were mechanically cleaved with scotch tape in air until the surface appeared flat and even. ZYH grade HOPG was selected to have a higher density of GBs in the samples. In synthetic crystals such HOPG, the GBs are expected to be confined to the basal plane, since non-basal dislocations (i.e., crossing many layers, like screw dislocations) can only occur in natural graphite crystals as an effect of the extreme mechanical strains occurring during rock formation [23]. The samples were loaded in a ultrahigh-vacuum (UHV) chamber (Omicron GmBH Multiprobe system VT-STM XA, base vacuum $5 \cdot 10^{-11} \mathrm{mbar}$ ) for STM measurements at room temperature. STM images were acquired using mechanically-created $\mathrm{Pt} / \mathrm{Ir}$ (20\% Ir) tips and electromechanically-etched $\mathrm{W}$ tips. The tips were conditioned until reaching atomic resolution both by electron bombardment, by fastscanning on flat terraces or by applying quick voltage pulses. A large number of images were acquired in various areas of the sample around GBs. All the STM images presented were acquired in constant current mode, using bias voltages in the range $1.2-1.5 \mathrm{~V}$ and current range $0.1-0.5 \mathrm{nA}$.

Molecular dynamics (MD) simulations were made with LAMMPS (Large-scale Atomic/Molecular Massively Parallel Simulator) [24]. Periodic boundary conditions were applied along the grain boundary direction. The unit cell was separated from its perpendicular images with a vacuum distance of $100 \mathrm{~nm}$ in the $\mathrm{z}$ direction. For each case, the cell length was $\sim 200 \mathrm{~nm}$, and the widths ranged from $10 \mathrm{~nm}$ up to $620 \mathrm{~nm}$. Adaptive Intermolecular Reactive Empirical Bond Order (AIREBO) potential from Stuart was used to simulate interaction among carbon atoms [25]. Structural energy minimum was achieved with the Polak-Ribière version of conjugate gradient algorithm [26], while simultaneously the lattice parameters were allowed to relax to obtain a net zero pressure on each face of the unit cell [27]. After this, a relaxation in a NVT ensemble was done for at least $1 \mathrm{~ns}$ with a time step of $10^{-3} \mathrm{ps}$ at $1 \mathrm{~K}$ of temperature. Finally, another structural energy minimum was found for the final relaxation.

For the simulation of different GB configurations, a graphene layer (with either a SGB or a DGB) on top of another rigid layer of graphene was modeled. Such graphene bilayer was made of two identical layers (the layers had $\mathrm{AB}$ stacking on one side only of the GB). The lower rigid layer was located $3.35 \AA$ away from the top one and its atoms were free to move only along the xy-plane, and not on the z-axis. This was meant to simulate the stiffness of a HOPG substrate underneath the top layer. The top layer atoms were completely free to move, with the exception of the atoms within $5 \AA$ from the edges, which were fixed in their position to avoid the influence of any other periodic image into the main unit cell. In the case of the DGB, two GBs running parallel were separated by a buffer region made of a (crystalline) graphene lattice, with a zigzag orientation parallel to the GBs.

To calculate the grain boundary energy per unit length as a function of the tilt angle, $E_{\text {total }}$ was obtained from our MD calculations and $E_{\text {bulk }}$ was the reference energy per atom calculated by MD for a pristine graphene with the same AIREBO potential. Although the exact positions of the carbon atoms at the buffer zone were unknown, we considered ordered graphene. This model represented a first approach where only the influence of the mismatch of the graphene sheets at the double grain boundary was considered. It was important to take into account the presence of the substrate, because it alters the height of the grain boundary with respect to suspended graphene. $A B$ stacking was chosen because it is a more stable and probable configuration than $\mathrm{AA}$ stacking.

\section{Results and discussion}

\subsection{STM measurements}

Numerous GBs are typically found on HOPG during STM measurements, exhibiting a wide variety of geometrical configurations. We have previously shown that a GB can give rise to large compression waves on a graphene sheet [17]. In the vast majority of cases, the edges between two graphene (or graphite) planes are merged by SGB, which can have different configurations, most often among three types: straight, sinuous or segmented. Experimental and theoretical studies $[3,28,29]$ showed that $(1,0)$ dislocations are typically the most stable defects forming GBs for $\theta \leq 21.8^{\circ}$. For higher angles, several complex configurations have been modeled, mainly formed by chains of $(1,0)$ and $(0,1)$ dislocations (i.e., the same kind of dislocations but with different orientation) $[3,28,29]$. The energetic equivalence of these two basic dislocations allows the two grains to merge by means of a sinuous and asymmetrical GB: in such cases, we speak of the formation of asymmetric bisector segments [30]. In our analysis, we found many of these structures, where the GB does not form a symmetric bisector between the planes. Some examples are reported in Fig. 1. A linear shape is generally found, regardless of $\theta$, in bisector GB, due to the classical dislocation theory in solids [8], which shows that identical edge dislocations are energetically favored to align. On the other hand, in a non-bisector GB a perfect alignment of all the dislocations is not possible. 

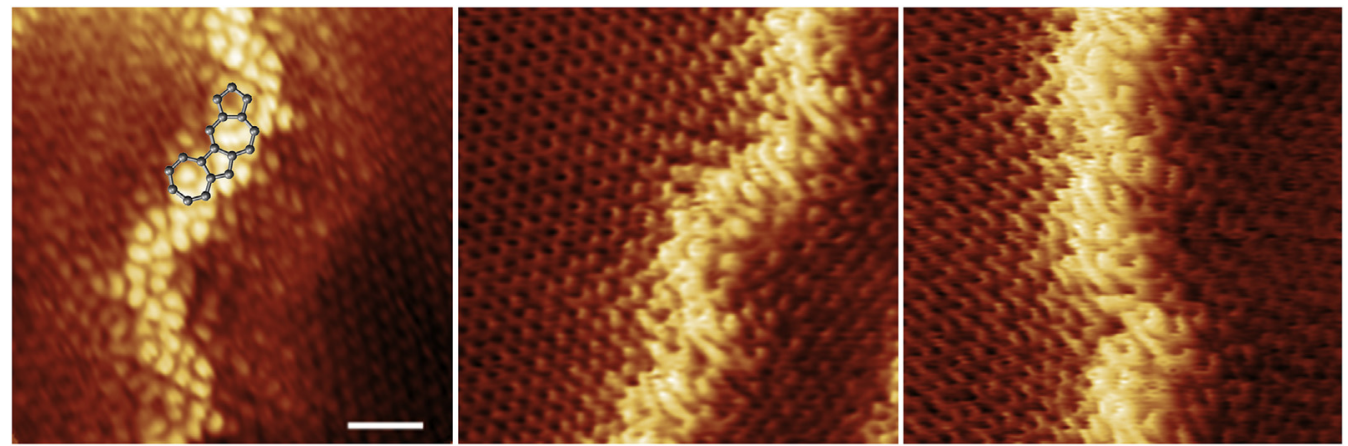

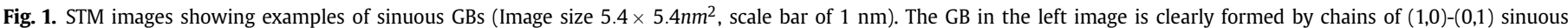

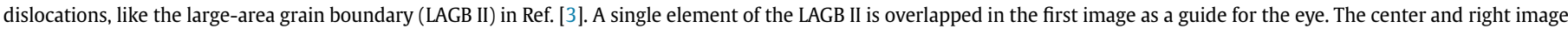
exhibit a less identifiable structure but still characterized by $(1,0)$ and $(0,1)$ dislocation sequences. (A colour version of this figure can be viewed online.)

Zhang et al. have proposed a possible mechanism of GB formation in these cases, where a repeated unit of a non-bisector GB is folded into two bisector segments, reducing the stress necessary for the formation of a straight GB [30]. Accordingly, in our experiments we observed a higher occurrence of sinuous GBs for increasing misorientation angles. We also observed often the occurrence of local superlattice effects, similar to those shown in Ref. [31]. These effects are due to line defect with charge enhancement, which is consistent with a series of atom vacancies or a split in the top graphene layer. They are usually visible in the immediate vicinity of the defect or appear as a moiré pattern in an adjacent area, as we report in Fig. S2 (image D4). In particular, an extended moiré pattern occurs only if the tilted graphite domain is localized not more than few layers below the top layer [31]. As mentioned before, defects with $22^{\circ}<\theta<35^{\circ}$ are typically modeled by complicated configurations which are generally less stable than low angle defects constituted by simple $(1,0)$ dislocation series. DFT calculations on graphene showed that the energy of a GB characterized by $\theta=$ $26^{\circ}$ can be $50 \%$ higher than that of a GB with $\theta=13^{\circ}[3,13]$, and similar considerations can be envisaged for the whole range $22^{\circ}<$ $\theta<35^{\circ}$. However, since all DFT calculations are performed at $\mathrm{T}=0 \mathrm{~K}$, it cannot be excluded that, under actual growth conditions at high temperature, DGBs can be stable in this range. We experimentally observed several configurations of DGBs, having the inner region between the two boundaries made of either an ordered or disordered atomic structure, as shown in Fig. 2. Two examples of ordered configurations with different shapes (straight and sinuous) are presented in Fig. 2a and b, while two disordered configurations are presented in Fig. 2c and d.

Our findings show that DGB can form between two graphite planes with $\theta>22^{\circ}$ (as we did not observe the occurrence of DGBs for lower $\theta$ in current and previous experiments) [17]. Particularly interesting is the occurrence, in several DGBs, of an inner region with ordered configuration. Fig. 3 reports an example of a DGB with $\theta=26.4^{\circ}$ with such ordered inner region. The double broken line along the two boundaries highlights the two parallel orientation $\theta_{1}$ and $\theta_{2}$. The angle between $\theta_{1}$ and $\theta_{2}$ is $120^{\circ}$, thus the GB edge is aligned with the zig-zag edge of the right graphite plane. The angle between $\theta_{2}$ and $\theta_{R}$ is $13.2^{\circ}$, corresponding to an ordered array of $(1,0)$ dislocations [29]. The angle between $\theta_{2}$ and $\theta_{L}$ is also exactly $13.2^{\circ}$, making the DGB symmetric with respect to the two planes. Fig. 3b shows the fast Fourier transform (FFT) of the STM image in Fig. 3a.

As expected, two sets of outer spots with hexagonal pattern (highlighted by different colors in Fig. 3b) correspond to the atomic arrangement of the two graphite planes. The ordered inner region of the DGB is testified by the pattern inside the two hexagons representing the left (yellow hexagon) and right (blue hexagon) planes. We were able to identify a coincidence lattice, with parallelogram symmetry and $4 \times 6$ periodicity (as referred to the graphite lattice parameter), that binds the supercell to the two planes on the left and right. The parallelogram angles are $82.2^{\circ}$ and $97.8^{\circ}$. Although the periodicity along the green lines in the FFT has not a straightforward interpretation (due to some disorder and to the zig-zag structure of the DGB), the vertical periodicity in the FFT is very clear. However, a careful comparison between the direct and reciprocal lattice allowed us to identify the supercell pointed out in Fig. 3.

To characterize the different DGB configurations, it is now worth to define another angle related to the orientation asymmetry of the graphite planes with respect to the DGB axis as $\psi=\left|\theta_{R}-\theta_{L}\right|$. In Fig. 4, we plotted a qualitative phase diagram reporting the different DGB configurations found in our STM analysis as a function of $\theta$ and $\psi$.

Our set of measurements excludes the occurrence of DGBs with ordered inner region configuration for $\theta>31^{\circ}$. This observation suggests an empirical limit for the formation of ordered DGB depending on $\theta$. Finally, we remark that disordered DGBs are generally not very sinuous. It is likely a disordered configuration is particularly efficient in relaxing the strain in the specific conditions of high $\psi$ and/or $\theta$ (i.e., low stability).

\subsection{MD simulations and total energy calculations}

Previous researches on MD have focused on the structural relaxation of a suspended graphene sheet with a SGB [3,26,32-36] and a DGB $[17,20,32,37]$ through the dependence of the GB energy per unit length: $\gamma=\left(E_{\text {total }}-n_{\text {atoms }} \cdot E_{\text {bulk }}\right) / l_{\mathrm{GB}}$, where $E_{\text {total }}$ is the net potential energy of the system, and $\mathrm{E}_{\mathrm{bulk}}=-7.807 \mathrm{eV}$ is the groundstate energy per atom in graphene calculated with the AIREBO potential. Other than being a theoretical support for our experimental results, our calculations also aim at complementing and extending the body of knowledge on the study of SGB and DGB in non-suspended graphene sheets (i.e. the most frequent situation for experiments and device fabrication). To achieve this, we modeled a graphene layer (with either a SGB or a DGB) on top of another rigid layer of graphene, in order to mimic HOPG, as shown in Fig. 5. To compare the experimental results to the MD simulations, two cases were built. The first case is a SGB between two graphene planes with $\theta=26^{\circ}$ (Fig. 5a). In this instance, the relaxation induces a strain release that lifts the GB by $2 \AA$ over the plane. The strain release zone is $\sim 4 \mathrm{~nm}$ wide. The second case is a DGB with an inner zigzag nanoribbon region (Fig. 5b). The DGB here has $\theta=13^{\circ}$ with respect to each graphene plane. The width of the 

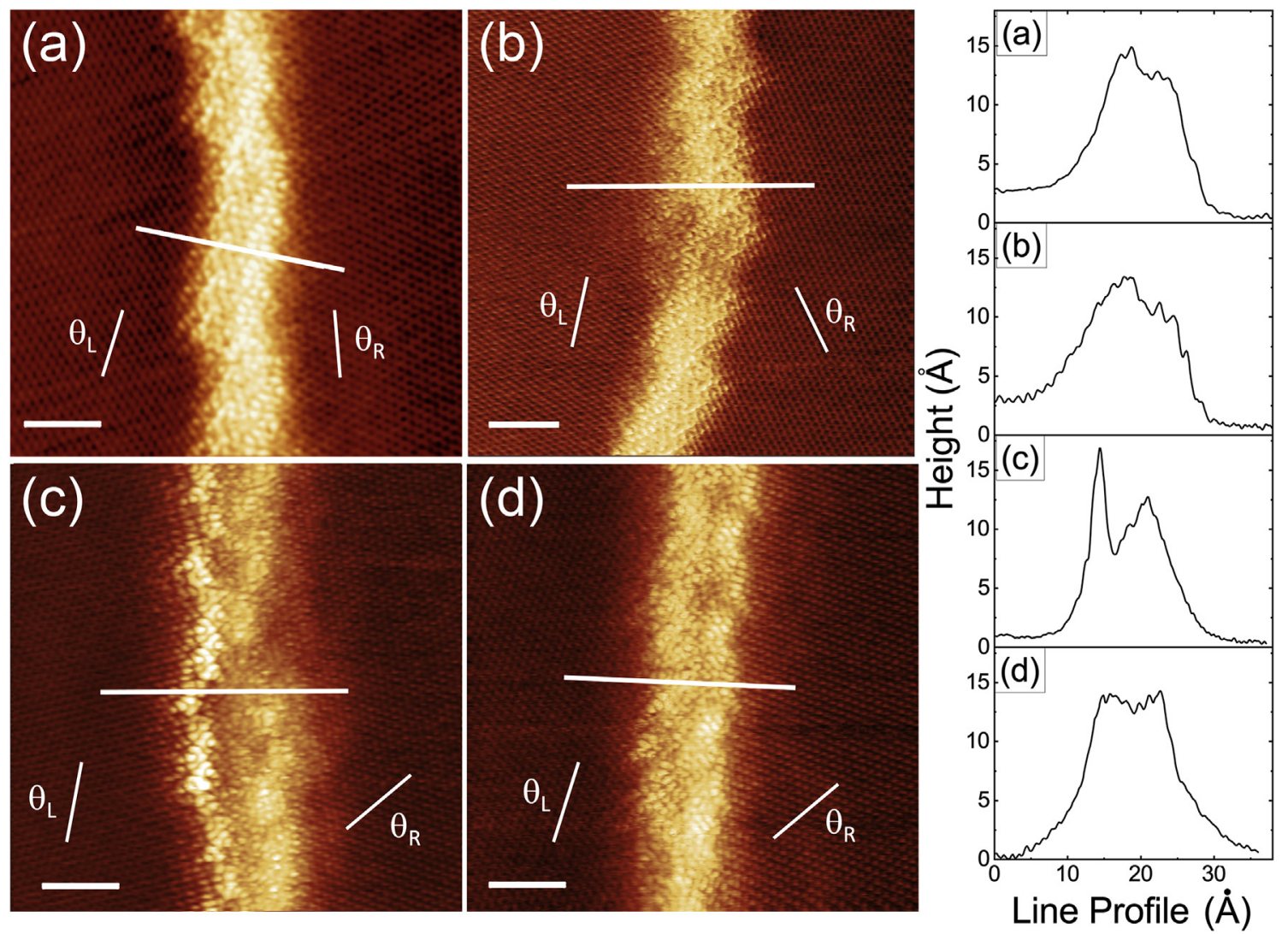

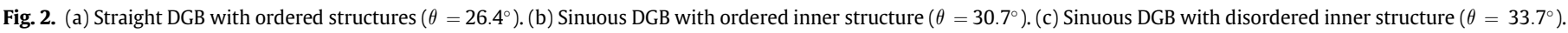

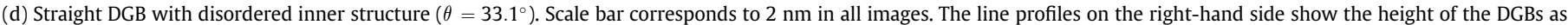
taken in each panel (by the white line), indicating values within 8-12 Å. (A colour version of this figure can be viewed online.)

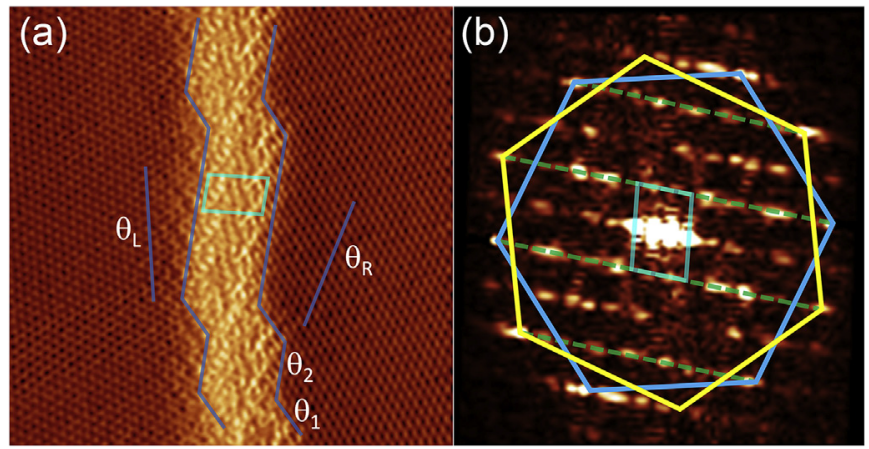

Fig. 3. (a) Zig-zag geometry of a straight ordered DGB (image size $6.5 \times 6.5 \mathrm{~nm}^{2}, \theta=$ $26.4^{\circ}$ ) and (b) corresponding FFT, highlighting the three ordered domains, as follows. The spots are arranged along two directions, which identify the orientation respectively in the left (yellow hexagon) and right (blue hexagon) regions, the inner spots are related to the region within the DGB and are related to the coincidence lattice with parallelogram symmetry and $4 \times 6$ periodicity that connect each vertex of the yellow hexagon to a corresponding vertex of the blue hexagon (as pointed out by dotted green lines). The $4 \times 6$ supercell is pointed out both in direct and reciprocal lattice by a cyan parallelogram. Further detail on the structure are presented in Fig. S1. (A colour version of this figure can be viewed online.)

embedded graphene ribbon is $\sim 2 \mathrm{~nm}$, which is comparable to what experimentally found. In this second case, the strain release is shared between the two GBs: although the maximum height measured are almost the same for SGB and DGB, there are more atoms raised from the graphene plane for the SGB (about $3.4 \%$ of total atoms in a $20 \times 20 \mathrm{~nm}$ sheet) than for the DGB (2\%). This result

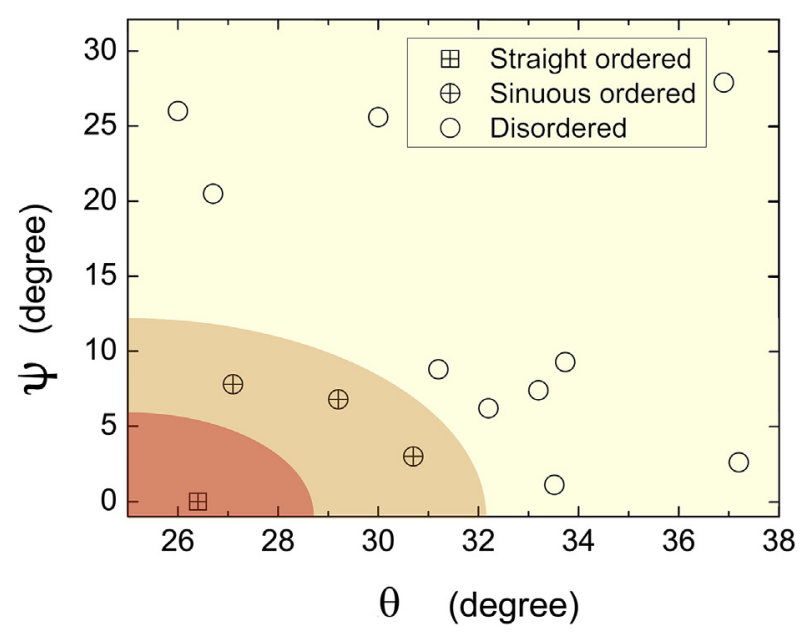

Fig. 4. Qualitative experimental phase diagram of the observed DGB nature as a function of $\theta$ and $\psi$. The boundaries between the different regions are qualitative. The disordered DGBs are not discriminated between straight and sinuous (as the ordered DGBs are) since their occurrence appeared random in our experimental observations. Fig. S2 reports the correspondence with each image. (A colour version of this figure can be viewed online.)

suggests that a double interface (i.e., the formation of a DGB) is a more efficient configuration to release the accumulated strain with respect to a single interface.

On average, the GB height profiles in our experimental data are

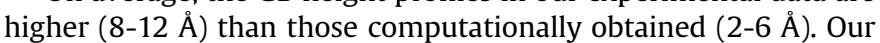



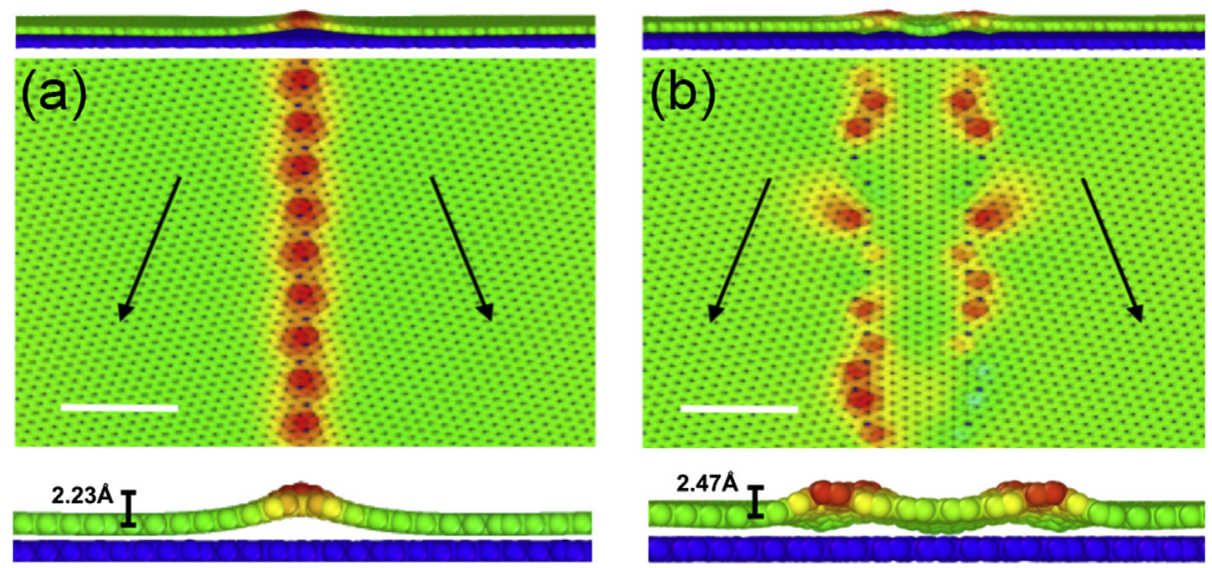

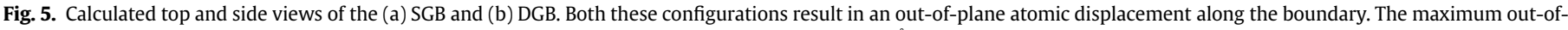

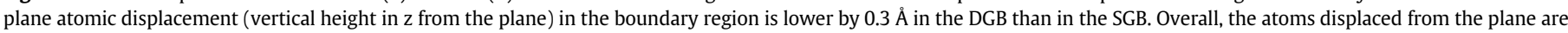
fewer in the DGB than SGB for the same area. Scale bar is $2 \mathrm{~nm}$. (A colour version of this figure can be viewed online.)

computational approach posited a graphene zigzag border parallel to the grain boundary, and this would serve as a very efficient strain release configuration, which would in turn lower the height of the atoms in the GB. Besides, the GB structures observed in our experiments are different and generally more complex than those posited in the computational models, and the same goes for the internal structure of the buffer zones.

In Fig. 6, the energy per unit length of the grain boundary is plotted as a function of $\theta$ for SGB and DGB for the case of a monolayer (ML) and a bilayer (BL). For $9^{\circ}<\theta<29^{\circ}$, the energy of the SGB and DGB have similar trends in all the range. Previous work by Carlsson et al. showed that in graphene there is an attractive interaction among neighboring GBs that can lower their formation energy in this range of misorientation angles [32]. As shown in Fig. 6, DGB and SGB have a similar energy trends in the vicinity of $26^{\circ}$, reaching a local minimum. This is in contrast with the calculations made on suspended graphene by Shekhawat et al. [35], which showed a local minimum at $22^{\circ}$ and a local maximum at around $26^{\circ}$. This difference between suspended (monolayer) and supported graphene (bilayer in our case) could be explained by the coupling between the top graphene layer and the rigid layer underneath. It should be also taken into account that this energy

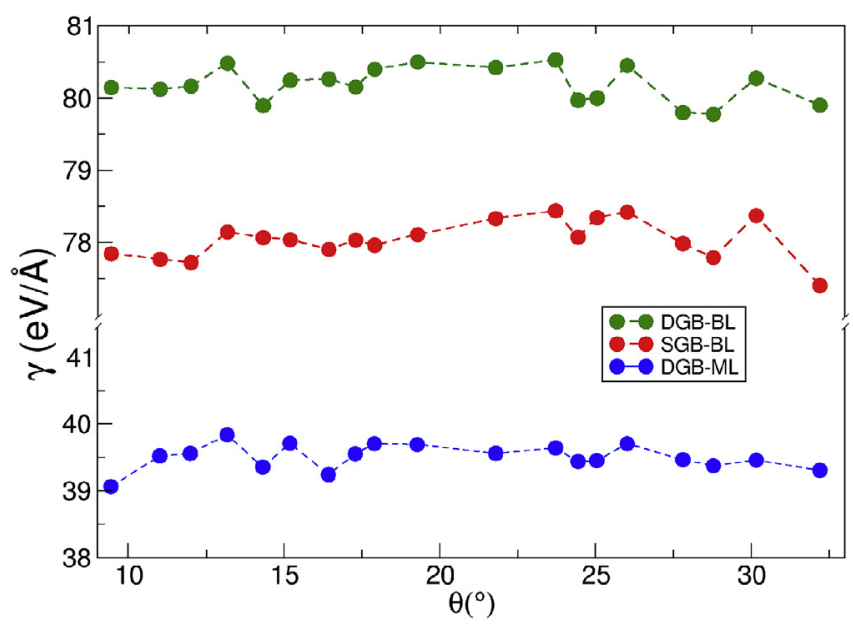

Fig. 6. Grain boundary energy per unit length as a function of the tilt angle for single grain boundary bilayer (SGB-BL), double grain boundary monolayer (DGB-ML) and bilayer (DGB-BL). (A colour version of this figure can be viewed online.) difference could be related as well to the stacking mismatch on one side of the boundary: If one side of the boundary is aligned with $A B$ stacking, the other side will have a mismatch between layers due to the relative angle of the two grains.

Although suspended graphene with $\theta=26^{\circ}$ is an unstable configuration, our results for supported graphene shows a local minima in energy at this angle. Meanwhile, the percentage of atoms raised above the plane for the DGB is less than for the SGB, proving that the DGB is more efficient in releasing the strain. Overall, the plots in Fig. 6 indicate that the relative energy differences between SGB-BL and DGB-BL is minor. Considering that the real conditions of the surfaces where the GBs appear are inherently different from the ideal conditions set in the simulations, the presence of a local minimum at $\theta \approx 26^{\circ}$ seems to confirm that around this angle there might exist the conditions for the formation of ordered DGBs, in line with the results of our experimental observations.

\section{Conclusions}

In summary, we have measured and analyzed the structure of grain boundaries on HOPG according to the misorientation angle between adjacent grains. On the one hand, for misorientation angles lower than $22^{\circ}$ we generally found the occurrence of SGBs, according to the literature. On the other hand, our STM observations point out that DGB structures can be stable for misorientation angles in the range $22^{\circ}-32^{\circ}$. In this range, the asymmetry angle (i.e., angle related to the orientation asymmetry of the graphite planes with respect to the DGB axis) dictates the configuration of the inner region between the two boundaries: an asymmetry angle higher than $10^{\circ}$ favors the formation of internal disorder, while lower values favor internal order (even with exact coincidence lattice superstructures). Notably, we systematically found internal disorder in DGBs with misorientation angles exceeding $32^{\circ}$. Therefore, our measurements detail several configurations of large angle grain boundaries, extending the experimental range of misorientation angles reported in literature. Our MD results and total energy calculations found a comparable stability between SGB and ordered DGB for misorientation angles between $22^{\circ}$ and $32^{\circ}$, corroborating the experimental evidence. Although the energy difference between the two configurations is small in this range, the total energy trend seems to always favor the formation of DGBs. Our experimental observations, as supported by the models, can be key for a detailed understanding of the angular requirements for the generation of complex grain boundaries structures, by 
providing grounds for further theoretical models and simulations. Our results provide an accurate determination of the energy and arrangement of these GBs, and could set the basis for a more extensive research on the production of controlled GBs for electronic and optoelectronic applications.

\section{Credit author contribution statement}

A. Capasso: conceptualization, methodology, experimental data acquisition and processing, writing (original draft), funding acquisition. F. Muñoz-Rojas: theoretical calculations and interpretation. B. Gupta: experimental data acquisition. N. Motta: conceptualization, data interpretation, funding acquisition. E. Placidi: conceptualization, methodology, experimental data acquisition and processing, writing (original draft). All authors contributed to the final writing.

\section{Declaration of competing interest}

The authors declare that they have no known competing financial interests or personal relationships that could have appeared to influence the work reported in this paper.

\section{Acknowledgements}

The authors acknowledge the financial support of the Australian Research Council through the LIEF grant (LE100100146). The technical support of Dr P. Hines of the Institute of Future environments at QUT and the Central Analytical Research Facility are kindly acknowledged. We also thank the Materials Science and Engineering Research Center HPC facility. A.C. acknowledges the support of the European Union's Horizon 2020 research and innovation program under the Marie Skłodowska-Curie grant agreement No. 713640 .

\section{Appendix A. Supplementary data}

Supplementary data to this article can be found online at https://doi.org/10.1016/j.carbon.2020.08.046.

\section{References}

[1] L. Gao, J.R. Guest, N.P. Guisinger, Epitaxial graphene on $\mathrm{Cu}(111)$, Nano Lett. 10 (9) (2010) 3512-3516.

[2] S.E. Krasavin, V.A. Osipov, Phonon scattering by externded defects in polycrystalline graphene, J. Struct. Chem. 59 (4) (2018) 860-866.

[3] O.V. Yazyev, S.G. Louie, Topological defects in graphene: dislocations and grain boundaries, Phys. Rev. B 81 (19) (2010).

[4] H.-L. Sun, Q.-T. Shen, J.-F. Jia, O--Z. Zhang, O.-K. Xue, Scanning tunneling microscopy study of superlattice domain boundaries on graphite surface, Surf. Sci. $542(1-2)(2003)$ 94-100.

[5] J. Cervenka, C.F.J. Flipse, Structural and electronic properties of grain boundaries in graphite: planes of periodically distributed point defects, Phys. Rev. B 79 (19) (2009)

[6] X. Feng, J. Wu, A.T. Bell, M. Salmeron, An atomic-scale view of the nucleation and growth of graphene islands on Pt surfaces, J. Phys. Chem. C 119 (13) (2015) 7124-7129.

[7] P.Y. Huang, C.S. Ruiz-Vargas, A.M. van der Zande, W.S. Whitney, M.P. Levendorf, J.W. Kevek, S. Garg, J.S. Alden, C.J. Hustedt, Y. Zhu, J. Park, P.L. McEuen, D.A. Muller, Grains and grain boundaries in single-layer graphene atomic patchwork quilts, Nature 469 (7330) (2011) 389-392.

[8] A. Luican-Mayer, J.E. Barrios-Vargas, J.T. Falkenberg, G. Autès, A.W. Cummings, D. Soriano, G. Li, M. Brandbyge, O.V. Yazyev, S. Roche, E.Y. Andrei, Localized electronic states at grain boundaries on the surface of graphene and graphite, 2D Mater. 3 (3) (2016), 031005.
[9] W.A. Diery, E.A. Moujaes, R.W. Nunes, Nature of localized phonon modes of tilt grain boundaries in graphene, Carbon 140 (2018) 250-258.

[10] P. Simonis, C. Goffaux, P.A. Thiry, L.P. Biro, P. Lambin, V. Meunier, STM study of a grain boundary in graphite, Surf. Sci. 511 (1-3) (2002) 319-322.

[11] P. Yasaei, A. Fathizadeh, R. Hantehzadeh, A.K. Majee, A. El-Ghandour, D. Estrada, C. Foster, Z. Aksamija, F. Khalili-Araghi, A. Salehi-Khojin, Bimodal phonon scattering in graphene grain boundaries, Nano Lett. 15 (7) (2015) $4532-4540$.

[12] J. Lahiri, Y. Lin, P. Bozkurt, I.I. Oleynik, M. Batzill, An extended defect in graphene as a metallic wire, Nat. Nanotechnol. 5 (5) (2010) 326-329.

[13] Z. Zhang, X. Zou, V.H. Crespi, B.I. Yakobson, Intrinsic magnetism of grain boundaries in two-dimensional metal dichalcogenides, ACS Nano 7 (12) (2013) 10475-10481.

[14] X. Zou, Y. Liu, B.I. Yakobson, Predicting dislocations and grain boundaries in two-dimensional metal-disulfides from the first principles, Nano Lett. 13 (1) (2012) 253-258.

[15] F. Gargiulo, O.V. Yazyev, Topological aspects of charge-carrier transmission across grain boundaries in graphene, Nano Lett. 14 (1) (2013) 250-254.

[16] Z. Song, V.I. Artyukhov, B.I. Yakobson, Z. Xu, Pseudo Hall-Petch strength reduction in polycrystalline graphene, Nano Lett. 13 (4) (2013) 1829-1833.

[17] A. Capasso, E. Placidi, H.F. Zhan, E. Perfetto, J.M. Bell, Y.T. Gu, N. Motta, Graphene ripples generated by grain boundaries in highly ordered pyrolytic graphite, Carbon 68 (2014) 330-336.

[18] K. Kim, Z. Lee, W. Regan, C. Kisielowski, M.F. Crommie, A. Zettl, Grain boundary mapping in polycrystalline graphene, ACS Nano 5 (3) (2011) $2142-2146$

[19] R. Grantab, V.B. Shenoy, R.S. Ruoff, Anomalous strength characteristics of tilt grain boundaries in graphene, Science 330 (6006) (2010) 946-948.

[20] B. Yang, S. Wang, Y. Guo, J. Yuan, Y. Si, S. Zhang, H. Chen, Strength and failure behavior of a graphene sheet containing bi-grain-boundaries, RSC Adv. 4 (97) (2014) 54677-54683.

[21] A.W. Tsen, L. Brown, M.P. Levendorf, F. Ghahari, P.Y. Huang, R.W. Havener, C.S. Ruiz-Vargas, D.A. Muller, P. Kim, J. Park, Tailoring electrical transport across grain boundaries in polycrystalline graphene, Science 336 (6085) (2012) 1143-1146.

[22] Z. Yang, F. Ma, K. Xu, Grain boundaries guided vibration wave propagation in polycrystalline graphene, RSC Adv. 7 (40) (2017) 24667-24673.

[23] C. Roscoe, J.M. Thomas, The revelation of small-angle boundaries and forest dislocations in graphite monocrystals, Carbon 4 (3) (1966) 383-390.

[24] S. Plimpton, Fast parallel algorithms for short-range molecular dynamics, J. Comput. Phys. 117 (1) (1995) 1-19.

[25] S.J. Stuart, A.B. Tutein, J.A. Harrison, A reactive potential for hydrocarbons with intermolecular interactions, J. Chem. Phys. 112 (14) (2000) 6472-6486.

[26] E. Polak, G. Ribiere, Note sur la convergence de méthodes de directions conjuguées, Revue française d'informatique et de recherche opérationnelle, Série rouge 3 (16) (1969) 35-43.

[27] M. Parrinello, A. Rahman, Polymorphic transitions in single crystals: a new molecular dynamics method, J. Appl. Phys. 52 (12) (1981) 7182-7190.

[28] J. Červenka, M.I. Katsnelson, C.F.J. Flipse, Room-temperature ferromagnetism in graphite driven by two-dimensional networks of point defects, Nat. Phys. 5 (11) (2009) 840-844.

[29] T.-H. Liu, G. Gajewski, C.-W. Pao, C.-C. Chang, Structure, energy, and structural transformations of graphene grain boundaries from atomistic simulations, Carbon 49 (7) (2011) 2306-2317.

[30] Z. Zhang, Y. Yang, F. Xu, L. Wang, B.I. Yakobson, Unraveling the sinuous grain boundaries in graphene, Adv. Funct. Mater. 25 (3) (2014) 367-373.

[31] H.S. Wong, C. Durkan, Imaging confined charge density oscillations on graphite at room temperature, Phys. Rev. B 84 (8) (2011), 085435.

[32] J.M. Carlsson, L.M. Ghiringhelli, A. Fasolino, Theory and hierarchical calculations of the structure and energetics of [0001] tilt grain boundaries in graphene, Phys. Rev. B 84 (16) (2011).

[33] M.D. Jiao, L. Wang, C.Y. Wang, Q Zhang, S.Y. Ye, F.Y. Wang, Molecular dynamics simulations on deformation and fracture of bi-layer graphene with different stacking pattern under tension, Phys. Lett. A 380 (4) (2016) 609-613.

[34] S. Malola, H. Häkkinen, P. Koskinen, Structural, chemical, and dynamical trends in graphene grain boundaries, Phys. Rev. B 81 (16) (2010).

[35] A. Shekhawat, C. Ophus, R.O. Ritchie, A generalized Read-Shockley model and large scale simulations for the energy and structure of graphene grain boundaries, RSC Adv. 6 (50) (2016) 44489-44497.

[36] X. Zhang, Z. Xu, Q. Yuan, J. Xin, F. Ding, The favourable large misorientation angle grain boundaries in graphene, Nanoscale 7 (47) (2015) 20082-20088.

[37] P. Hirvonen, M.M. Ervasti, Z. Fan, M. Jalalvand, M. Seymour, S.M. Vaez Allaei, N. Provatas, A. Harju, K.R. Elder, T. Ala-Nissila, Multiscale modeling of polycrystalline graphene: a comparison of structure and defect energies of realistic samples from phase field crystal models, Phys. Rev. B 94 (3) (2016). 